\title{
Black Tea Extract Attenuates Sodium Fluoride-Induced Neurotoxicity in Adult Male Rats
}

\author{
Adeoye 0 Oyewopo ${ }^{1 *}$ Samuel K Olaniyi ${ }^{2}$ and Omotola M Oyeleke ${ }^{2}$ \\ ${ }^{1}$ Department of Anatomy, University of Ilorin, Nigeria \\ ${ }^{2}$ Department of Physiology, Afe Babalola University, Nigeria
}

*Corresponding author: Adeoye O Oyewopo, College of Health Sciences, Department of Anatomy, University of Ilorin P.M.B.1515 Ilorin, Nigeria, Tel: +2348033925431; Email: wolesake@yahoo.com

\section{Research Article \\ Volume 2 Issue 1}

Received Date: February 06, 2018

Published Date: February 20, 2018

\section{Abstract}

Background: Excessive exposure to fluoride has been documented to cause brain injury and reduce intelligence quotient even in school children. Black tea which has flavonoid as its highest constituent has been reported as antioxidant, antiinflammatory, antitumor, antimutagenic and ant carcinogenic. However, the neuroprotective effect of black tea is ill defined. We therefore hypothesize that black tea extract would attenuate sodium fluoride-induced neurotoxicity.

Method: Adult male Wistar rats were randomly allotted into groups; Control (vehicle; received distilled water), sodium fluoride-treated (NaF-treated; received $10 \mathrm{mg} / \mathrm{kg} \mathrm{b.w)}$, Black tea extract-treated (BTE-treated; received 2\% black tea extract) and sodium fluoride+ Black tea extract-treated (NaF+BTE -treated). The treatment lasted for 28 days and the administration was done daily by oral gavage. The body weight change was monitored using animal weighing balance (Olympia SCL66110 model, Kent Scientific Corporation, Torrington, CT06790, USA), biochemical assay and cerebellar tissue histology were performed as previously described.

Results: The results showed significant increase in body weight gain and significant cerebellar degeneration in NaFtreated group when compared with the vehicle-treated group. These alterations of cerebellar morphology were associated with decreased cerebellar glucose-6-phophate dehydrogenase, lactate dehydrogenase, and glutathione peroxidase. However, Concomitant administration of black tea extract during treatment with NaF significantly attenuated cerebellar degeneration.

Conclusion: The present study demonstrates that administration of black tea extract during treatment with $\mathrm{NaF}$ preserves cerebellar function, suggesting that possibly daily intake of BTE prevents the onset of neurodegenerative disorders. This might be due to its antioxidative effect.

Keywords: Antioxidant; Black Tea; Cerebellum; Intelligent quotient; Sodium fluoride 


\section{Journal of Natural \& Ayurvedic Medicine}

Abbreviations: ROS: Reactive Oxygen Species, LPO: Lipid Peroxidation, F: Fluoride (F), BBB: Blood Brain Barrier, BTE: Black Tea Extract

\section{Introduction}

Fluorine, a toxic and reactive element, is widely prevalent throughout the environment and it is a natural component of the earth's crust and occurs in varying concentration in rocks, soil, water and air. It is present in several chemical forms either organic or inorganic, one of the commonest inorganic fluorides is sodium fluoride [1]. Like many elements, it is beneficial to human health in trace amounts, but can be toxic in excess [2]. Excessive intake of fluoride $(\mathrm{F})$ has been well documented to cause fluorosis, which is a progressive degenerative disorder that predominantly affects the skeletal system, teeth, neuronal structure and function in animals and humans $[3,4]$. Earlier studies have shown a correlation between fluoride exposure and the intelligence quotient of school children and have also suggested that children with dental fluorosis are at greater risk of impaired mental acuity [3].

In addition, number of studies has clearly shown that $\mathrm{F}$ increases formation of brain reactive oxygen species (ROS) and lipid peroxidation (LPO) activity. Excessive ingestion of Fluoride (F) can exert toxic effects on many tissues and organs, resulting in serious damage and pathological changes. A number of histopathological changes, including demyelination, decrease in the number of Purkinje cells, thick and disappearance of dendrites, swelling of mitochondria and dilation of endoplasmic reticulum in neurons, have been observed in the brain of experimental animals subjected to fluorosis [5,6]. Also accumulation of $F$ has been observed in the brains of experimental animals exposed to high doses of $\mathrm{F}$ for a prolonged period, which can eventually cross the blood brain barrier (BBB). Alteration in the BBB can also promote inflammation involving activated microglia which may be preferentially associated with the amyloid plaques, these capillary distortions can disrupt blood flow patterns, altering cerebral metabolism and if sustained, contribute to progressive degeneration of neurons [7]. Fluoride's damage to neurons can reflect both an increase in oxidative processes and a decrease in antioxidant defenses. Fluorosis patients may undergo neuro degeneration by fluoride induced-oxidative stress [6] However, the effects of fluoride compounds on human health have received worldwide attention because of their environmental persistence, industrial exposure and widespread distribution. Its neurotoxicity is well proven clinically but, the detailed histological changes have not been fully studied and it is not well recognized if these neurological symptoms could be prevented or minimized by protective agents.

Antioxidants are capable of stabilizing, or deactivating free radicals before they attack cells and they are absolutely critical for maintaining optimal cellular and systemic health and well-being [8]. To overcome free radical mediated consequences of disease process and drug therapies, various antioxidant supplements have been proposed and have been shown to play an important role in cellular protection [8]. Reports indicate that administration of antioxidants with L-DOPA therapy protect against ROS-induced damage both in vivo and in vitro [9]. The progressive oxidative stress-induced neurodegeneration is not halt/slow down by the currently used drug therapies. Hence, current researches are focusing on finding therapies, preferentially natural products including food supplements which could help in preventing/delaying the ongoing oxidative stress-induced neurodegeneration $[10,11]$. Recently, a novel dietary strategy was proposed that is specifically tailored toward lowering the risk of human neurodegeneration, which includes eating a nutritionally balanced diet that contains adequate amounts of fruits and vegetables, along with adequate dietary supplementation of vitamins C, B6, B12 and folate [12]. Further, supplementation of dietary antioxidants such as adenosine, selenium, $\alpha$-tocopherol, ascorbic acid, Nacetyl cysteine, tea polyphenols and flavonoids have been shown to protect against oxidative stress and exert neuroprotective action [13-15]. Among these, tea and tea polyphenols have attracted increasing interest because besides their radical scavenging property [16], they have also been shown to have iron chelating, anti inflammatory, antiapoptotic, antineoplastic, anticarcinogenic, antimutagenic and antiangiogenic properties under in vivo and in vitro condition as well as the ability to modulate cell signaling [17-23]. Moreover, tea has been shown to possess stronger antioxidant property than typical antioxidants such as glutathione, ascorbic acid, $\alpha$-tocopherol and than that of most reported dietary agents on a daily basis [24]. Besides black tea has an added advantage of being one of the most commonly worldwide consumed beverages. Its constituents include catechins, theaflavins, thearubigins and flavonoids and have the ability to penetrate BBB and fulfill the requirement of a potential neuroprotective agent $[12,17,25]$. Further, tea extract and polyphenols have been shown to attenuate MPTP and 6-OHDAinduced cell death in vivo and in vitro studies $[23,26]$. Research till date, has been mainly focused on green tea, whereas there is not much information available exploring the neuroprotective potential of black tea, the consumption of which amounts to be $80 \%$ of total tea consumption worldwide $[27,28]$. In addition, it has been reported that in comparison to green tea, black tea has an 


\section{Journal of Natural \& Ayurvedic Medicine}

advantage of being highly rich in multimeric polyphenols (theaflavins), generated during tea leaves fermentation, which is reported to be strongest antioxidant among catechins [29-30]. Black tea has been shown to be more effective iron chelator, thus preventing metal catalyzed free radical formation [29]. In the present investigation, an attempt has been made to study the structural changes which might result from sodium fluoride on the cerebellar cortex of adult male rats and to evaluate whether black tea extract was able to reduce or improve these changes.

\section{Materials and Methods}

\section{Chemicals}

The entire chemicals used in the study were of AR grade, which were available locally. Dry black tea leaves were purchased from Ogbomoso market.

\section{Preparation of the extract}

Aqueous black tea extract (BTE) was freshly prepared every day. For preparing 2\% BTE, $2 \mathrm{~g}$ of BT leaves were suspended in $100 \mathrm{ml}$ of hot water $\left(85^{\circ} \mathrm{C}\right)$, brewed for 5 $\mathrm{min}$, cooled to room temperature and filtered.

\section{Animals, Grouping and protocol}

Twenty adult male Wistar rats weighing 200-230g were obtained from the animal house, College of Medicine and Health Sciences, Ladoke Akintola University of Technology, Ogbomoso, Oyo State, Nigeria. The rats were housed in wire mesh cages and maintained in a well ventilated room at $25 \pm 2{ }^{\circ} \mathrm{C}$, on a 12 -h light/12-h dark cycle. Rats had unrestricted access to standard rat chow and tap water. After acclimatized for two weeks, the rats were randomly allotted into groups $(\mathrm{n}=5$ each); Control (vehicle; received distilled water), sodium fluoridetreated (NaF-treated; received $10 \mathrm{mg} / \mathrm{kg}$ b.w), Black tea extract-treated (BTE-treated; received 2\% black tea extract) and sodium fluoride+ Black tea extract-treated (NaF+BTE -treated). The administration of $\mathrm{NaF}$ was done daily by oral gavage while rats were given $2 \%$ BTE ad libitum to drink instead of water. The treatment lasted for 28 days. The investigation was conducted in accordance with the National Institutes of Health Guide for the Care and Use of Laboratory Animals and was approved by the Institutional Review Board of Ladoke Akintola University of Technology, Ogbomoso, and every effort was made to minimize both the number of animals used and their suffering. Initial and final body weights were monitored using animal weighing balance (Olympia SCL66110 model, Kent Scientific Corporation, Torrington, CT06790, USA) and the body weight gain was estimated.

\section{Sample Preparation and Biochemical Analysis}

At the end of treatment, the rats were anesthetized with pentobarbital sodium (50 mg/kg, i.p). Blood was collected from the apex of the heart into heparinized bottle and centrifuged at $3000 \mathrm{rpm}$ for 15 minutes using a bench centrifuge and the plasma was stored frozen until it was needed for biochemical assay. Biochemical analysis of plasma malondialdehyde (MDA) was performed using assay kit. The cerebella were excised, blotted and weighed. After weighing, 500mg of tissue was carefully removed and homogenized with a glass homogenizer. Following centrifugation at 3000rpm for 10 minutes, supernatant was removed and used for the measurement of Glucose-6- Phosphate Dehydrogenase (G6PDH), Lactate Dehydrogenase (LDH), and Glutathione Peroxidase (GPX) activities by standardized enzymatic colorimetric methods using assay kit obtained from Randox Laboratory Ltd. (Co. Antrim, UK).

\section{Histology}

The cerebella were excised, blotted and weighed. After weighing, the cerebellar tissues were fixed in $10 \%$ buffered normal saline for histological examination using hematoxylin and eosin (H\&E) staining technique. The tissues were dehydrated by passing ascending grades of alcohol through them, clearing them in xylene, and finally embedding them in paraffin wax (mp $56^{\circ} \mathrm{c}-58^{\circ} \mathrm{c}$ ). Transverse sections were cut on a microtome. These sections were stained with Ehrlich's hematoxylin and eosin in alcohol, dehydrated in alcohol, cleared in xylene, mounted in DPX, and examined microscopically.

\section{Statistical Analysis}

All data were expressed as means \pm SEM. Statistical group analysis was performed with SPSS, version 22 of statistical software. One-way analysis of variance (ANOVA) was used to compare the mean values of variables among the groups. Bonferroni's test was used to identify the significance of pair wise comparison of mean values among the groups. Statistically significant differences were accepted at $p<0.05$.

\section{Results}

\section{Effect of BTE on Body Weight in NaF-Treated Adult Male Rats}

Table 1 depicts the effect of administration BTE and $\mathrm{NaF}$ on body weight. The results showed no significant change in body weight gain of NaF- or NaF+BTE-treated groups compared with control group. However, a significant increase $(p<0.05)$ was observed in the body weight gain of BTE-treated group compared with control group. 


\section{Journal of Natural \& Ayurvedic Medicine}

\begin{tabular}{|c|c|c|c|}
\hline Groups & $\begin{array}{c}\text { Initial } \\
\text { b.w(g) }\end{array}$ & $\begin{array}{c}\text { Final } \\
\text { b.w.(g) }\end{array}$ & $\begin{array}{c}\text { B.W. } \\
\text { gain (g) }\end{array}$ \\
\hline Control & $227.8 \pm 12.9$ & $246.5 \pm 13.7$ & $18.7 \pm 2.8$ \\
\hline NaF-treated & $221.5 \pm 15.4$ & $231.5 \pm 12.7$ & $10.0 \pm 5.9$ \\
\hline BTE-treated & $205.8 \pm 10.4$ & $255.3 \pm 11.9$ & $49.5 \pm 10.2 *$ \\
\hline NaF+BTE-treated & $223.6 \pm 16.8$ & $251.9 \pm 18.2$ & $28.3 \pm 7.2$ \\
\hline
\end{tabular}

Data are expressed as mean \pm S.E.M. $n=5$. Data were analysed by one-way ANOVA followed by Bonferroni post hoc test. $\left({ }^{*} p<0.05\right.$ vs control).

Table 1: Effect of BTE on body weight in NaF-treated adult male rats.

\section{Effect of BTE on the Histology of Cerebellum in NaF-Treated Adult Male Rats}

Histopathological changes in the cerebella have been reported to affect the functionality of this salient part of the brain. $\mathrm{H} \& \mathrm{E}$ section of the cerebellar cortex of control and BTE-treated groups showed the well known normal structure. They showed three distinct layers from outside inwards; the molecular layer, the mono layer of Purkinje cells and the closely packed granular cell layer. The Purkinje cells were arranged in one row between the molecular and granular layers. The granular layer was composed of closely packed numerous small granular cells with dark nuclei Figure 1a,c. Examination of the photomicrograph of a section of cerebellar cortex in NaFtreated rat revealed multifocal neuronal affection especially on the Purkinje cell layer. The monolayer arrangement of Purkinje cells was disrupted in many areas, as they disappeared completely in some areas while they revealed multilayer deposition with loss of the normal shape. The molecular and granular layers showed slight disruption and there was a decreased number of white matter compared with control group Figure $1 \mathrm{~b}$. Also, examination of the photomicrograph of a section of cerebellar cortex in NaF+BTE-treated rat showed monolayer arrangement of Purkinje cells with mild disorganization, mild variation in molecular and granular layers compared with control and in addition revealed an improved structure compared with NaF-treated group Figure 1d.
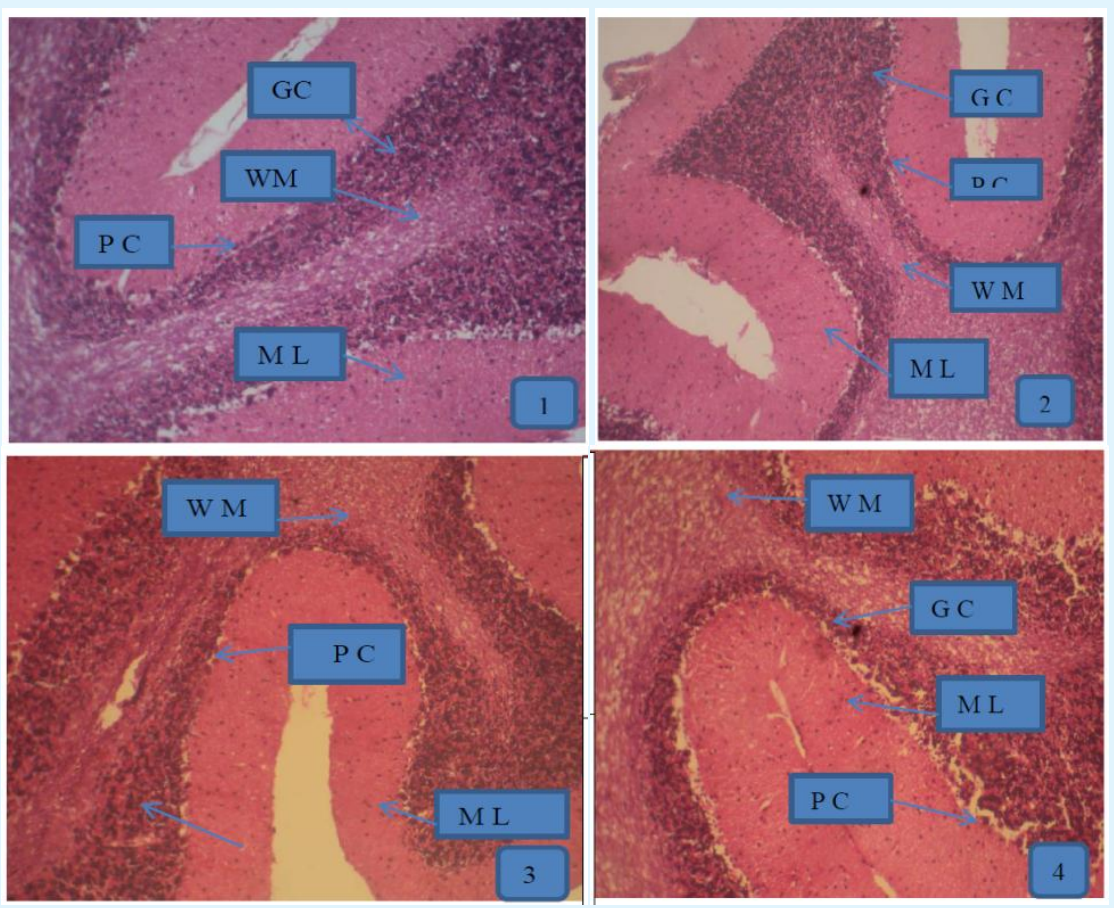

Figure 1: Photomicrographs of a section of cerebellum in control- (a), NaF- (b), BTE- (C) and NaF+BTE-treated rats (d). ML (molecular layer); GC (Granular cells); PC (Purkinje cells); WM (White matter). Control rat, shows normal molecular layer, closely packed granular cell layer, mono layer of purkinje cells and normal white matter, NaF-treated rat, shows disrupted layer of purkinje cells and disappearance of purkinje cell layer in some areas, slight disruption of molecular and granular layers and decreased number of white matter, BTE-treated rat, shows normal molecular layer, closely packed granular cell layer and mono layer of purkinje cells and NaF+BTE-treated rat, shows mono layer arrangement of purkinje cells with mild disorganization, normal white matter, mild variation in molecular and granular layers compared with control. (H \& E paraffin stain; $\times 200$, transverse section). 


\section{Journal of Natural \& Ayurvedic Medicine}

\section{Effect of BTE on Oxidative Stress Marker in NaF-Treated Adult Male Rats}

Plasma MDA is a potent biomarker of oxidative stress. Treatment with $\mathrm{NaF}$ significantly induced oxidative stress with significant increased level of plasma MDA when compared with control group. However, concomitant administration of BTE during treatment with $\mathrm{NaF}$ prevented oxidative stress with a significant decreased level of plasma MDA when compared with NaF-treated group Figure 2.

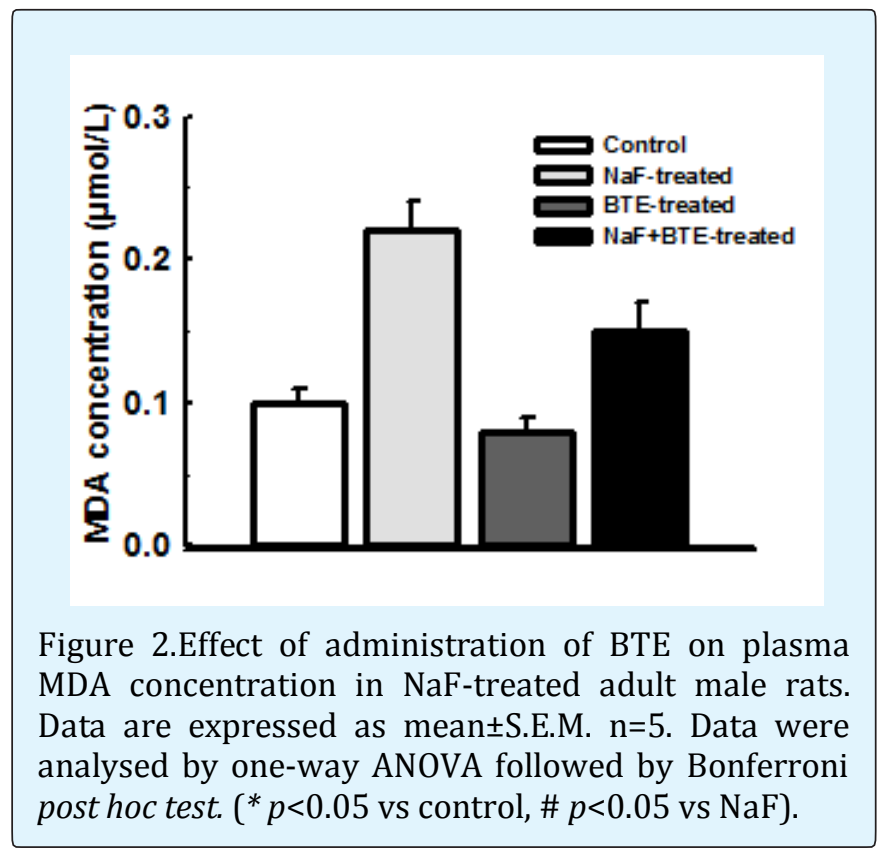

\section{Effect of BTE on Cerebellar Endogenous Defense Mechanism in NaF-Treated Adult Male Rats}

Glucose-6- Phosphate dehydrogenase, Lactate dehydrogenase and Glutathione peroxidase activities are bioindicators of cellular defense mechanism. Exposure to $\mathrm{NaF}$ significantly reduced G6PDH, LDH and GPX activities compared with control group. However, administration of BTE during treatment with $\mathrm{NaF}$ significantly preserved the cellular endogenous defense mechanism with increased activities of G6PDH, LDH and GPX significantly except GPX when compared with NaF-treated group Figure 3a, b, c.

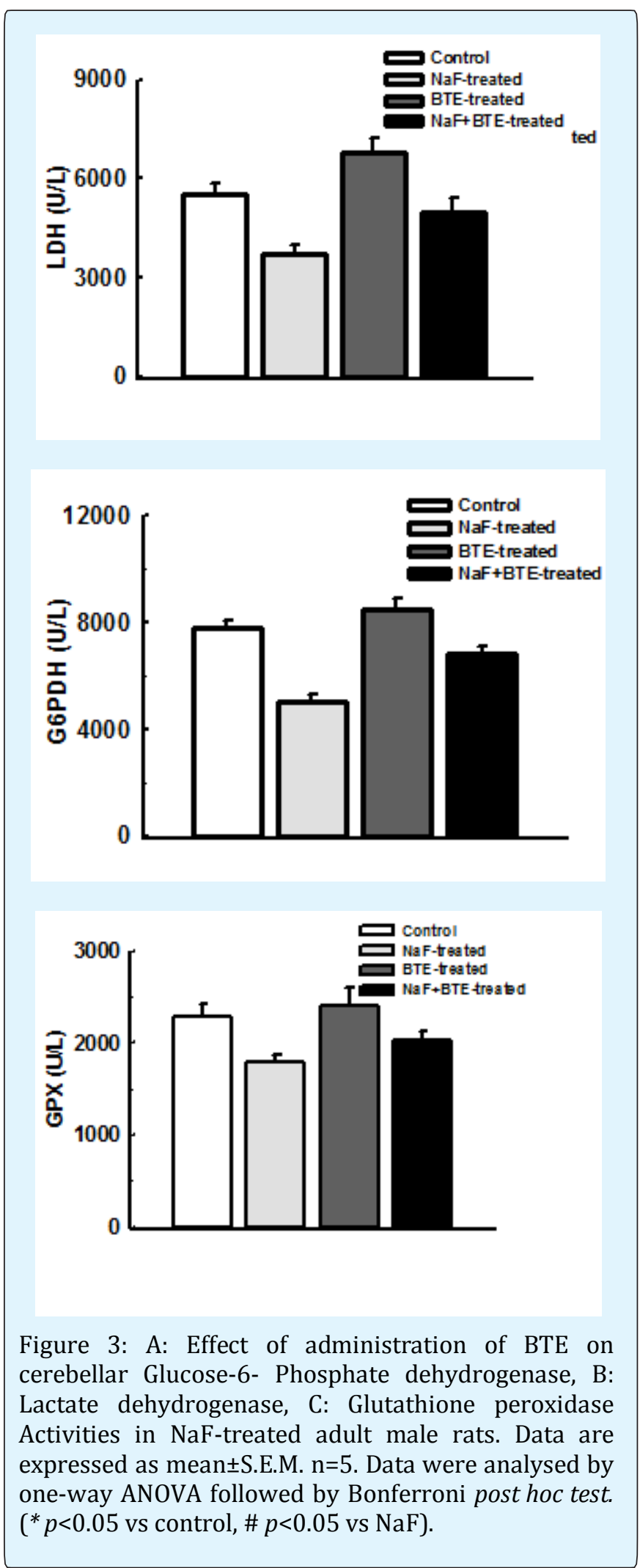




\section{Journal of Natural \& Ayurvedic Medicine}

\section{Discussion}

The current study has attempted to investigate the neurotoxic and neuroprotective effects of $\mathrm{NaF}$ and BTE respectively in animal model. Our results demonstrate that chronic exposure to $\mathrm{NaF}$ did not significantly alter body weight but led to significant disruption of purkinje cell layer, slight disruption of molecular and granular layers with decreased number of white matter in the histology of cerebellar cortex. These variations in the cellular arrangement of the cerebellar cortex were associated with significant increase in plasma MDA level, significant decrease in cerebellar G6PDH, LDH and GPX activities when compared with control group. In addition, administration of BTE during treatment with $\mathrm{NaF}$ significantly preserved the monolayer arrangement of purkinje cells, molecular and granular layers with normal proportion of white matter in the histology of cerebellar cortex. These observations were also associated with significant decrease in plasma MDA level, significant increase in cerebellar G6PDH and LDH activities when compared with NaF-treated group. However, cerebellar GPX of NaF+BTE-treated group did not significantly increased when compared with NaF-treated group and likewise the cellular arrangement of cerebellar cortex showed mild variations when compared with control group. Plasma MDA, cerebellar G6PDH, LDH and GPX activities were not significantly different from the control group.

Fluoride is unavoidably and uncontrollably introduced into the body through fluoridation of drinking water and treatment of dental caries. Its undesirable adverse effects have been reported [3-4]. Fluoride is completely and quickly absorbed from the gastrointestinal tract and affects many organs [31]. A few studies related to the neurotoxic effect of chronic oral ingestion of $\mathrm{NaF}$ have been documented [6,12]. Our present results that chronic exposure to $\mathrm{NaF}$ significantly alters the cellular arrangement of cerebellar cortex is consistent with previous studies that oral ingestion of $\mathrm{NaF}$ resulted to neurological abnormality and selective structural changes in the brain [6]. The pathological changes in the structure of cerebellar cortex following the administration of $\mathrm{NaF}$ impaired cerebellar function, which corroborates earlier observation that oral ingestion of $\mathrm{NaF}$ led to neurological changes and subsequently resulted to cerebellar ataxia that manifests muscle weakness, tremors with movement, loss of equilibrium, gait disturbance and hind limbs paralysis $[32,6]$. Another previous study confirmed that ingested fluoride could be retained by the cerebellum, interfering with its physiology and inducing neurotoxicity, cell damage and even cell death [33]. This study revealed that $\mathrm{NaF}$ induced structural changes and disorganization in Purkinje cells. Their arrangement was disrupted; some disappeared completely while in other areas they exhibited multilayer accumulation. This might be caused by displacement from some areas to be accumulated in others. Most of them lost their shape and showed irregular outline. Similar findings were earlier reported that chronic fluoride intoxication caused marked neurodegenerative changes in rats and impaired mental acuity in humans especially in the early stages of life $[34,3]$. In addition, these changes may form the neural basis for impaired learning, memory and abnormal behaviour patterns that have been earlier reported and disturbed overall body physiology [3].

Many investigators have attributed the structural and neuropathological changes that resulted from $\mathrm{NaF}$ ingestion to pathophysiological mechanisms like inhibition of $\mathrm{Na}+\mathrm{K}+-\mathrm{ATP} a \mathrm{se}$, leading to extracellular release of potassium with subsequent hyperkalamia. In addition, fluoride ions chelate calcium leading to hypocalcaemia and interference with calcium function [35]. Subsequently, it had been known that fluoride induced inflammatory reaction, cell cycle arrest and apoptosis in different systems. Also, it showed diverse actions on a variety of cellular and physiological functions including inhibition of a variety of enzymes as metalloenzymes that were proved to be involved in many essential metabolic processes as glycolysis, oxidative phosphorylation and neuro-transmission [36].

However, the mechanisms underlying the neurotoxic effect of endemic fluorosis still remain unclear. Our current results revealed that the altered cerebellar structure and function in NaF-treated animals was associated with significant increase in plasma level of MDA (Figure 2). Increased concentration of circulating MDA (oxidative stress marker) observed in the present study is an indication of oxidative stress, which implies that $\mathrm{NaF}$-induced cerebellar degeneration is mediated by oxidative stress. This finding provides further evidence to previous studies that increased MDA concentration induces lipid peroxidation of cellular membrane which damages the cell plasma membrane and results in cell death, and thus, high lipid peroxidation alters cerebellar structure and function $[37,38]$. This observation was also consistent with previous report that fluoride induced significant increase in intracellular reactive oxygen species (ROS), malondialdehyde levels and the percentage of apoptosis [3,39]. Which means NaF could impact its detrimental effect via oxidative stress, which could lead to apoptosis, decreased mRNA and protein expression levels of neural cell adhesion molecules in rat neurons, contributing to the neuronal dysfunction and brain injury [40]. The current results also revealed that administration of $\mathrm{NaF}$ significantly induced decrease in cerebellar G6PDH, LDH and GPX activities. This implies that NaF 


\section{Journal of Natural \& Ayurvedic Medicine}

could trigger the activity of G6PDH in order to release NADPH. G6PDH is critical in the regeneration of NADPH a co-enzyme that is essential in protection against and repairs of oxidative damage and also plays a major role in maintaining the proper 3-dimentional structure of proteins in the cell membranes [41]. As the first and ratelimiting enzyme in the pentose phosphate pathway the role of G6PDH becomes critical in the integrity of the neurons as the entire antioxidant system and other processes requiring reduction rely on the adequate supply of NADPH. A decrease in the G6PDH activity and subsequent decrease in NADPH makes the cell very susceptible to oxidative stress. In addition, a significant reduction in LDH activity during treatment with $\mathrm{NaF}$ indicates an imbalance between anaerobic and aerobic glucose metabolism in the brain which could lead to lactate accumulation as previously reported [42] and this could be a contributing factor to cerebellar structural and functional disruption. The findings that GPX activity significantly reduces, suggests a reduction in cellular defense against oxidative damage making the rats susceptible to oxidative stress. Glutathione is the major endogenous antioxidant produced by the cells, participating directly in the neutralization of free radicals and reactive oxygen compounds, as well as maintaining exogenous antioxidant in their reduced forms [18]. Glutathione serves as protective mechanism whereby potentially toxic electrophilic metabolites are "mopped up" as glutathione conjugates [43]. A significant decrease in the cerebellar GPX activity that is observed in the current study indicates an altered cerebellar cellular redox status. This also confirmed that the cerebellar tissue disruption observed in the current study is associated with oxidative stress.

In the current study, the concomitant administration of BTE and NaF attenuated/reduced the signs of neurotoxicity caused by $\mathrm{NaF}$ and revealed mild neurotoxic effect on the Purkinje cells of adult male rats. The structure and function of cerebellar cortex were significantly preserved in NaF+BTE-treated rats. This implies that BTE, which main components are catechins, theaflavins, thearubigins and flavonols elicits neuroprotective effect against NaF-induced neurotoxicity. The absence of any morphological change in the cerebellar cortex of rats treated with BTE alone confirmed that BTE elicits neuroprotective effect (Figure 1c). In addition, the findings of the present study revealed that the neuroprotective effect of BTE against NaF-inducedneurotoxicity was associated with significant decrease in circulating level of MDA (Figure 2). This implies that BTE elicits its neuroprotective action via regulation of oxidative stress markers (MDA). The significant decreased level of MDA in BTE-treated alone when compared with control group also confirmed BTE as a potent mitigant of oxidative stress. Our present result was consistent with earlier study that black tea was shown to restore the level of brain oxidative markers against alcohol intoxication [25]. The current study also observed a significant increase in cerebellar tissue defense mechanism with the administration of BTE during treatment with $\mathrm{NaF}$ which was evident with significant increased in cerebellar G6PDH and LDH activities when compared with NaF-treated group. This is an indication that BTE is a potent modulator of antioxidant enzymes to elicit its neuroprotective action against oxidative stress induced-neurotoxicity. This observation was in consonance with earlier study that BTE components possess strong antioxidant and iron-chelating properties that are involved in neuroprotection against 6-OHDA model of Parkinson disease in rats [44]. The effectiveness of BTE as a promising neuroprotective agent can be attributed to its ability to penetrate the blood brain barrier [25] and mop up toxic electrophilic metabolites which are capable of causing cellular disruption and apoptosis. To the best of our knowledge the results of this study suggest for the first time that administration of BTE during treatment with $\mathrm{NaF}$ preserves the structure and function of cerebellar cortex, thus prevents neurodegeneration $[45,46]$.

\section{Conclusion}

The current study suggests that chronic exposure to $\mathrm{NaF}$ causes neurotoxic effects as evident with cerebellar cortex disruption, perhaps via increased oxidative stress. However, concomitant administration of BTE attenuates the neurotoxic effects.

\section{Conflict of Interest}

The authors declare that there are no conflicts of interest.

\section{References}

1. Pessan JP, Silva SM, Lauris JRP, Sampaio FC, Whitford GM, et al. (2008) Fluoride uptake by plaque from water and from dentifrice. J Dent Res 87(5): 461-465.

2. Fordyce FM, Vrana K, Zhovinsky E, Povoroznuk V, Toth G, et al. (2007) A health risk assessment for fluoride in Central Europe. Environ Geochem Health 29(2): 83-102.

3. Trivedi MH, Verma RJ, Chinoy NJ, Patel RS, Sathawara NG (2007) Effects of high fluoride water on intelligence of school children in India. Fluoride 40(3): 178-183. 


\section{Journal of Natural \& Ayurvedic Medicine}

4. Verma RJ, Guna SDM (2000) Sodium fluoride-induced hypoproteinemia and hypoglycemia in parental and $\mathrm{F}$ (1)-generation rats and amelioration by vitamins. Food Chem Toxicol 40(12): 1781-1788.

5. Varner JA, Jensen KF, Horvath W, Isaacson RL (1998) Chronic administration of aluminum-fluoride or sodiumfluoride to rats in drinking water: alterations in neuronal and cerebrovascular integrity. Brain Res 784(1-2): 284-292.

6. Sharma C, Pooja S, Piyu S, Neha J, Maheep B (2014) Curcumin attenuates neurotoxicity induced by fluoride: An in vivoevidence. Pharmacogn Mag 10(37): 61-65.

7. Vander Voet GB, Schijns O, De Wolff FA (1999) Fluoride enhances the effect of aluminium chloride on interconnections between aggregates of hippocampal neurons. Arch Physiol Biochem 107(1): 15-21.

8. Mattson MP, Duan W, Chan SL, Cheng A, Haughey N, et al. (2002) Neuroprotective and neurorestorative signal transduction mechanisms in brain aging: modification by genes, diet and behavior. Neurobiol Aging 23(5): 695-705.

9. Chalimoniuk M, Stepien A, Strosznajder JB (2004) Pergolide mesylate, a dopaminergic receptor agonist, applied with l-DOPA enhances serum antioxidant enzyme activity in Parkinson disease. Clin Neuropharmacol 27(5): 223-229.

10. Dawson TM, Dawson VL (2002) Neuroprotective and neurorestorative strategies for Parkinson's disease. Nat Neurosci 5: S1058-S1061.

11. Pan M, Lai C, Wang H, Lo C, Ho C, et al. (2013) Black tea in chemo-prevention of cancer and other human diseases. Food Science and Human Wellness 2(1): 1221.

12. CS Reddy N, Pratap Reddy K (2016) Aluminum and fluoride impacts cortex and hippocampus structure in rats: protective role of resveratrol. International Journal of Applied Biology and Pharmaceutical Technology 8(1): 89-97.

13. Soto-Otero R, Mendez-Alvarez E, Hermida-Ameijeiras A, Munoz- Patino AM, Labandeira Garcia JL (2000) Autoxidation and neurotoxicity of 6hydroxydopamine in the presence of some antioxidants: potential implication in relation to the pathogenesis of Parkinson's disease. J Neurochem 74(4): 1605-1612.
14. Roghani M, Behzadi G (2001) Neuroprotective effect of vitamin E on the early model of Parkinson's disease in rat: behavioral and histochemical evidence. Brain Res 892(1): 211-217.

15. Sweeney MI, Kalt W, MacKinnon SL, Ashby J, Gottschall-Pass KT (2002) Feeding rats diets enriched in lowbush blueberries for six weeks decreases ischemia-induced brain damage. Nutr Neurosci 5(6): 427-431.

16. Mercer LD, Kelly BL, Horne MK, Beart PM (2005) Dietary polyphenols protect dopamine neurons from oxidative insults and apoptosis: investigations in primary rat mesencephalic cultures. Biochem. Pharmacol 69(2): 339-345.

17. Rah DK, Han DW, Baek HS, Hyon SH, Park JC (2005) Prevention of reactive oxygen species-induced oxidative stress in human microvascular endothelial cells by green tea polyphenol. Toxicol Lett 155(2): 269-275.

18. Pan HH, Suda Y, Ohe Y, Sumi M, Yoshioka M (2002) Effect of aspartame on methyl-D-aspartate-sensitive $\mathrm{L}-[3 \mathrm{H}]$ glutamate binding sites in rat brain synaptic membranes. Brain Res 520(1-2): 351-353.

19. Steele VE, Kelloff GJ, Balentine D, Boone CW, Mehta R, et al. (2000) Comparative chemo preventive mechanisms of green tea, black tea and selected polyphenols extracts measured by in vitro bioassays. Carcinogenesis 21(1): 63-67.

20. Lambert JD, Hong, J, Yang GY, Liao J, Yang CS (2005) Inhibition of carcinogenesis by polyphenols: evidence from laboratory investigations. Am J Clin Nutr 8(1S): S284-S291.

21. Halder B, Pramanick S, Mukhopadhyay S, Giri AK (2005) Inhibition of benzo[a]pyrene induced mutagenicity and genotoxicity by black tea polyphenols theaflavins and thearubigins in multiple test systems. Food Chem Toxicol 43(4): 591-597.

22. Oak MH, El Bedoui J, Schini-Kerth VB (2005) Antiangiogenic properties of natural polyphenols from red wine and green tea. J Nutr Biochem 16(1): 18.

23. Levites Y, Amit T, Youdim MB, Mandel S (2002) Involvement of protein kinase $\mathrm{C}$ activation and cell survival/cell cycle genes in green tea polyphenols epigallocatechin 3 gallate neuroprotective actions. J Biol Chem 277(34): 30574-30580. 


\section{Journal of Natural \& Ayurvedic Medicine}

24. Rechner AR, Wagner E, Van Buren L, Van De Put F, Wiseman S, et al. (2002) Black tea represents a major source of dietary phenolics among regular tea drinkers. Free Radic Res 36(10): 1127-1135.

25. Luczaj W, Skrzydlewska E (2004) Antioxidant properties of black tea in alcohol intoxication. Food Chem Toxicol 42(12): 2045-2051.

26. Nie G, Cao Y, Zhao B (2002) Protective effects of green tea polyphenols and their major component, epigallocatechin-3-gallate (EGCG), on 6hydroxydopamine-induced apoptosis in PC12 cells. Redox Rep 7(3): 171-177.

27. Ames BN, Shigenaga MK, Hagen TM (1993) Oxidants, antioxidants, and the degenerative diseases of aging. Proc Natl Acad Sci USA 90(17): 7915-7922.

28. Ahmad N, Katiyar SK, Mukhtar H (1998) Cancer chemoprevention by tea polyphenols. Nutrition and chemical toxicity 10: 301-343.

29. Dufresne CJ, Farnworth ER (2001) A review of latest research findings on the health promotion properties of tea. J Nutr Biochem 12(7): 404-421.

30. Leung LK, Su Y, Chen R, Zhang Z, Huang Y, et al. (2001) Theaflavins in black tea and catechins in green tea are equally effective antioxidants. J Nutr 131(9): 2248-2251.

31. Amira S, Soufane S, Gharzouli K (2005) Effect of sodium fluoride on gastric emptying and intestinal transit in mice. Exp Toxicol Pathol 57(1): 59-64.

32. Fitzgerald MJT, Folan Curran J (2002) Clinical neuroanatomy and related neuroscience. $5^{\text {th }}$ (Edn.), WB Saunders.

33. Trabelsi M, Guermazi F, Zeghalc N (2001) Effect of fluoride on thyroid function. Fluoride 34(3):165-173.

34. Shivarajashankara YM, Shivashankara AR, Bhat PG, Rao SM, Rao SH (2002) Histological changes in the brain of young fluoride-intoxicated rats. Fluoride 35(1): 12-21.

35. Boink AB, Wemer J, Meulenbelt J, Vaessen HA, De Wildt DJ (1994) The mechanism of fluoride-induced hypocalcaemia. Hum Exp Toxicol 13(3): 149-155.

36. Refsnes M, Thrane EV, Lag M, Hege Thoresen G, Schwarze PE (2001) Mechanisms in fluoride-induced interleukin-8 synthesis in human lung epithelial cells. Toxicology 167(2): 145-158.
37. Ogeturk M, Kus I, Colakoglu N, Zararsiz I, Ilhan N, et al. (2005) Caffeic acid phenethyl ester protects kidneys against carbon tetrachloride toxicity in rats. J Ethnopharmacology 97(2): 273-280.

38. Karandez A, Yildirim A, Celebi F (2007) Protective effect of panax ginseng against carbon tetrachloride $\left(\mathrm{CCl}_{4}\right)$-induced oxidative brain injury in rats. Atatürk Üniversitesi Vet Bil Derg 2(3): 117-121.

39. Ling Y, Shengnan L, Chen Wang F, Wang YS, Nan Yan SX, et al. (2013) JNK and NADPH Oxidase Involved in Fluoride-Induced Oxidative Stress in BV-2 Microglia Cells, Mediators Inflamm 895975: 1-10.

40. Zhang M, Wang A, Xia T, He P (2008) Effects of fluoride on DNA damage, S-phase cell cycle arrest and the expression of $\mathrm{NF}-\mathrm{\kappa B}$ in primary cultured rat hippocampal neurons. Toxicol Lett 179(1): 1-5.

41. Bocchetta A (2003) Psychotic mania in glucose-6phosphate dehydrogenase-deficient subjects. Ann Gen Hosp Psychiatry 2(1): 6-15.

42. Schurr A, Payne RS (2007) Lactate, not pyruvate is neuronal aerobic glycolysis end product: An invitro electrophysiological study. Neuroscience 147(3): 613-619.

43. Scholz RW, Graham KS, Gumpricht E, Reddy CC (1989) Mechanism of interaction of interaction of vitamin $\mathrm{E}$ and glutathione in the protection against membrane lipid peroxidation. Ann NY Sci 570: 514517.

44. Pinto NB, Alexandre BD, Tavares Neves KR, Silva AH, Leal LKA, et al. (2015) Neuroprotective properties of the standardized extract from camellia sinensis (green tea) and its main bioactive components, epicatechin and epigallocatechin gallate, in the 6ohda model of parkinson's disease. Evidence-Based Complementary and Alternative Medicine, Article ID 161092, 12 pages.

45. Arab L, Il'yasova D (2003) The epidemiology of tea consumption and colorectal cancer incidence. J Nutr 133(10): 3310-3340.

46. Zhu BT (2004) CNS dopamine oxidation and catecholO-methyltransferase: importance in the etiology, pharmacotherapy, and dietary prevention of Parkinson's disease. Int J Mol Med 13(3): 343-353. 